\title{
Julia Geschke
}

\section{Das reformierte Unterhaltsprivileg}

Im Zusammenhang mit der Frage der Verfassungsmäßigkeit von $§ 32$ VersAusglG 
WISSENSCHAFTLICHE BEITRÄGE AUS DEM TECTUM VERLAG

Reihe Rechtswissenschaften 
https://doi.org/10.5771/9783828870697-|

Generiert durch IP '172.22.53.54', am 26.04.2023, 10:13:25.

Das Erstellen und Weitergeben von Kopien dieses PDFs ist nicht zulässig. 


\section{WISSENSCHAFTLICHE BEITRÄGE AUS DEM TECTUM VERLAG}

Reihe Rechtswissenschaften

Band 117

Julia Geschke

Das reformierte Unterhaltsprivileg

Im Zusammenhang mit der Frage der

Verfassungsmäßigkeit von § 32 VersAusglG

Tectum Verlag 
Julia Geschke

Das reformierte Unterhaltsprivileg. Im Zusammenhang mit der Frage der Verfassungsmäßigkeit von § 32 VersAusglG

Wissenschaftliche Beiträge aus dem Tectum Verlag:

Reihe: Rechtswissenschaften; Bd. 117

(C) Tectum - ein Verlag in der Nomos Verlagsgesellschaft, Baden-Baden 2019

Zugl. Diss. Christian-Albrechts-Universität zu Kiel 2017

E-Book: 978-3-8288-7069-7

ISSN: $1861-7875$

(Dieser Titel ist zugleich als gedrucktes Werk unter der ISBN

978-3-8288-4097-3 im Tectum Verlag erschienen.)

Alle Rechte vorbehalten

Besuchen Sie uns im Internet

www.tectum-verlag.de

Bibliografische Informationen der Deutschen Nationalbibliothek Die Deutsche Nationalbibliothek verzeichnet diese Publikation in der Deutschen Nationalbibliografie; detaillierte bibliografische Angaben sind im Internet über http://dnb.d-nb.de abrufbar. 
Gewidmet meinem Mann Felix und meiner Familie. 
https://doi.org/10.5771/9783828870697-|

Generiert durch IP '172.22.53.54', am 26.04.2023, 10:13:25.

Das Erstellen und Weitergeben von Kopien dieses PDFs ist nicht zulässig. 


\section{Inhaltsverzeichnis}

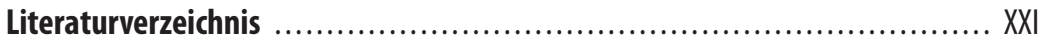

Einleitung - Gegenstand, Ziel und Gang der Untersuchung ................. 1

1. Kapitel: Grundlagen - der Versorgungsausgleich im 21. Jahrhundert ..... 7

§ 1 Die bis 2009 geltende Rechtslage ............................ 7

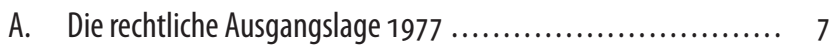

I. Intention des damaligen Gesetzgebers ................... 8

II. Grundgedanke bei der rechtlichen Ausgestaltung ......... 10

B. Bedeutende Veränderungen in den vergangenen 30 Jahren ..... 11

I. Gesellschaftliche und wirtschaftliche Entwicklung ......... 11

II. Schwächen der bis 2009 geltenden gesetzlichen Ausgestaltung .................................... 12

1. Probleme im Rahmen der "Einmalsaldierung“ .......... 12

2. Probleme im Rahmen der Durchführung ............... 14

III. Stellungnahme zur defizitären Lage .................... 16

§ 2 Verfassungsrechtlicher Hintergrund des Versorgungsausgleichs der alten Rechtslage ......................................... 17

A. Rechtliche Beurteilung des BVerfGs im Grundsatzurteil $1980 \ldots 17$

I. Inhalt des Grundsatzurteils ......................... 17

II. Umsetzung durch den Gesetzgeber .................... 19

III. Stellungnahme ......................................... 21

B. Weitere verfassungsrechtlich relevante Themen des alten

Rechts ........................................... 23

I. Entscheidung aus dem Jahr 1986 zum schuldrechtlichen Versorgungsausgleich ................................ 23 
II. Zum Hintergrund des Abänderungsverfahrens gem.

$\S 10 a$ VAHRG

1. Gründe für die Einführung ........................... 24

2. Entscheidung des BVerfGs aus dem Jahr 1993 .......... 25

3. Stellungnahme zur Einführung des $\S 10 a$ VAHRG a.F. .... 26

C. Exkurs: Zusätzliche Gesetzesergänzungen des

Versorgungsausgleichsrechts

§ 3 Die Neugestaltung des Versorgungsausgleichs im Rahmen der Strukturreform 28

A. Gesetzgeberische Ausgangslage ......................... 28

B. Der Weg des neuen Versorgungsausgleichsgesetzes ........... 29

C. Zielsetzungen des Gesetzgebers .......................... 30

I. Zusammenfassung der Reformgründe ................... 37

1. Verfehlung der Halbteilung durch die Einmalsaldierung

a. Die problematische Umsetzung im Rahmen der Durchführung

b. Nachträglich eintretende Änderungen bezogen auf den Ausgleich

2. Zunehmende Bedeutung der ergänzenden Altersversorgung ................................. 32

II. Bestrebungen des Gesetzgebers ...................... 33

1. Beseitigung des Gerechtigkeitsdefizits ................ 33

2. Beseitigung des Anwendungsdefizits ................. 33

a. Neue Gestaltungsspielräume für Ehegatten und Gerichte ................................... 35

b. Entlastungen der Versorgungsträger ............. 35

$\S 4$ Die Neuerungen des Versorgungsausgleichs im Einzelnen ........... 35

A. Materiell-rechtliche Änderungen .............................. 35

I. Funktion und dogmatische Einordnung des neuen Versorgungsausgleichs .............................. 36

II. Grundzüge des geltenden Rechts ..................... 37

1. Die neue Ausgleichsform .......................... 37

a. Wertausgleich bei der Scheidung ............... 37

b. Ausgleichsansprüche nach der Scheidung ........ 38

2. Auszugleichende Anrechte ........................ 39 
3. Bestimmung von Ehezeit und Ehezeitanteil 40

4. Ausnahmen von der Durchführung des Wertausgleichs 41

a. Kein Ausgleich wegen Geringfügigkeit

b. Kein Ausgleich bei fehlender Ausgleichsreife gem. $\S 19$ VersAusglG

c. Kein Ausgleich wegen unbilliger Härte gem. § 27 VersAusglG

5. Erweitere Spielräume für Ehegatten

6. Wegfall des Rentner- und Pensionistenprivilegs und der Regelungen über den Höchstbetrag

B. Formale Neustrukturierung der Korrekturmöglichkeiten

I. Die Härtefallregelungen als Anpassungen nach Rechtskraft

II. Die Totalrevision als neues Abänderungsverfahren im neuen FamFG 46

C. Verfahrensrechtliche Neuerungen im Zusammenhang mit dem FamFG 48

I. Die Scheidung als verfahrensrechtlicher Ausgangspunkt .... 48

1. Grundsätzliches zum FamFG 48

2. Verfahrensrechtliche Einteilung des Scheidungsverfahrens im FamFG

II. Die Versorgungsausgleichssachen als gewöhnliche Familiensachen 53

1. Verfahrensrechtliche Auswirkungen 53

2. Exkurs: Einordnung der Unterhaltssachen i.S.v. § 111 Nr. 8 FamFG

III. Die Versorgungsausgleichssachen im Zusammenhang mit dem Verbund

1. Die Besonderheiten von Folgesachen im Rahmen des Verbundes 55

2. Die Versorgungsausgleichssachen als Folgesachen 57

a. Unterscheidung innerhalb der Versorgungsausgleichsverfahren 57

b. Verfahrensgrundsätze des Verbundes 58

c. Die Abtrennung vom Verbund gem. $§ 140$ Abs. 2 FamFG 
IV. Verfahren in Versorgungsausgleichssachen

1. Verfahren in Versorgungsausgleichssachen gem. § 217 FamFG 59

2. Verfahrensregelungen gem. $\S \S 217 \mathrm{ff}$. FamFG 61

a. Umfassender Erörterungstermin nach $§ 221$ FamFG 61

b. Zu den verfahrensrechtlichen Auskunftspflichten gem. § 220 FamFG .......................... 63

c. Aussetzung des Verfahrens in Versorgungsausgleichssachen .................. 63

$\S 5$ Fazit 64

2. Kapitel: Die Regelung des $\S 32$ VersAusgIG ............................. 65

$\S 1$ Vergleich der Rechtslagen .................................... 65

A. Der Anwendungsbereich von $\S 32$ VersAusg|G i.R.d. Anpassungsverfahren ....................................... 65

I. Der Anwendungsbereich der alten Rechtslage ............ 65

1. Erweiterung durch $\S 10$ VAHRG a.F. ................. 66

a. Anrechte i.R.d. Ausgleichs nach $\S 1$ Abs. 3 VAHRG a.F. ....................................... 66

b. Anrechte i.R.d. Ausgleichs nach $\S 1$ Abs. 2 VAHRG a.F. ...................................... 67

aa. Privatrechtlich organisierter Versorgungsträger ...................... 68

bb. Öffentlich-rechtlicher Versorgungsträger ..... 69

2. Erweiterung durch $\S 3 b$ Abs. 1 VAHRG a.F. i.V.m. $\S 10$ VAHRG a.F. ...................................... 70

3. Zwischenergebnis .............................. 71

II. Der neue Anwendungsbereich i.S.v. § 32 VersAusglG ....... 71

III. Modifizierungen durch $\S 32$ VersAusg|G .................. 74

1. Vergleich der Rechtslagen ........................... 74

2. Ausführungen des Gesetzgebers .................... 76

3. Gegenmeinung ................................. 77

4. Stellungnahme .................................. 79

5. Zwischenergebnis ................................. 80 
B. Der Anwendungsbereich von $\S 32$ VersAusglG i.R.d.

Abänderungsverfahren

80

I. Der uneingeschränkte Anwendungsbereich von § 10a

VAHRG a.F. ......................................... 80

II. Einschränkungen durch $\S 32$ VersAusg|G ................. 81

1. Vergleich der Rechtslagen ......................... 81

2. Ausführungen des Gesetzgebers ..................... 83

3. Gegenmeinung ............................... 84

4. Stellungnahme ….............................. 86

5. Zwischenergebnis ............................... 87

C. Ergebnis .................................................. 87

§ 2 Verfassungsrechtliche Untersuchung ........................... 87

A. Verfassungsrechtlicher Diskurs i.R.d. Anpassungsverfahren ..... 88

I. Verfassungsrechtliche Relevanz nach Ansicht des BVerfGs ... 89

1. Entscheidung des BVerfGs aus dem Jahr 2014 .......... 89

2. Vergleich zur bisherigen Rechtsprechung des BVerfGs ... 90

3. Stellungnahme ................................ 92

a. Feststellungen zum Verstoß des $\S 32$ VersAusglG gegen Art. $14 \mathrm{GG}$............................. 92

b. Feststellungen zum Verstoß des $\S 32$ VersAusgIG gegen Art. 3 Abs. 1 GG ........................ 94

4. Ergebnis .................................... 95

II. Verfassungsrechtliche Diskussion i.R.d. Anpassungsverfahren ............................... 96

1. Keine verfassungsrechtlichen Bedenken ............... 96

a. Verstoß gegen das Versicherungsprinzip ......... 97

b. Erhöhter Stellenwert der Versorgungen der Regelsicherungssysteme ..................... 98

c. Unterschiedliche Struktur der Regelsicherungssysteme ...................... 98

d. Keine Drittwirkung der Grundrechte ............. 99

2. Verfassungsrechtliche Bedenken - Gegenansicht ....... 99

a. Überbewertung des Versicherungsprinzips ........ 101

b. Gestiegene Bedeutung der ergänzenden Altersversorgung ............................. 101

c. Keine Unterscheidung bei der Eigentumsgarantie in Art. 14 Abs. $1 \mathrm{GG}$ 
d. Kein verfassungswidriger Eingriff bei privaten Versorgungsträgern ........................... 104

3. Stellungnahme .................................... 106

a. Bewertung des Versicherungsprinzips ............. 106

b. Gestiegene Bedeutung der ergänzenden Altersversorgung .............................. 106

c. Keine Unterscheidung bei der Eigentumsgarantie ............................ 107

d. Abwägung der Grundrechtspositionen privater Versorgungsträger ............................... 108

e. Ergebnis ................................. 108

4. Eröffnung des Regelungsbereichs durch entsprechende Anwendung? 109

a. Keine entsprechende Anwendung auf andere Zusatzversorgungen ........................... 110

b. Gegenmeinung ............................... 111

c. Stellungnahme .............................. 112

B. Verfassungsrechtlicher Diskurs i.R.d. Abänderungsverfahren .... 114

I. Keine verfassungsrechtlichen Bedenken ................... 114

II. Die Gegenmeinung - verfassungsrechtliche Bedenken ...... 115

III. Stellungnahme ....................................... 117

C. Fazit ................................................... 119

3. Kapitel: Das reformierte Unterhaltsprivileg .......................... 121

$\S 1$ Einleitender Teil ......................................... 122

A. Zweck und Wirkung des Unterhaltsprivilegs ................. 122

B. Die Bestrebungen des Gesetzgebers bei der Neugestaltung im Rahmen der Strukturreform ................................ 123

I. Begrenzung der Aussetzung auf die Höhe des Unterhalts ... 124

II. Zuständigkeit der Familiengerichte ...................... 125 
§ 2 Darstellung der Rechtslage 126

A. Materiell-rechtliche Regelungen gem. § 33 VersAusglG 126

I. Grundtatbestand des $\S 33$ Abs. 1 VersAusglG 126

1. Alte Rechtslage: Voraussetzungen gem. $\S 5$ Abs. 1 VAHRG a. F.

a. Bezug einer Versorgung des Ausgleichspflichtigen 127

b. Kein Rentenanspruch des Ausgleichsberechtigten 128

c. Nachehelicher Unterhaltsanspruch 130

aa. Bestehen eines Unterhaltsanspruchs 130

bb. Vorliegen eines gesetzlichen Unterhaltsanspruchs

cc. Prüfungsumfang der Versorgungsträger

dd. Bindung an bereits bestehende Unterhaltstitel? 133

d. Rechtsfolge

2. Ausführungen des Gesetzgebers 135

3. Neue Rechtslage gem. $\S 33$ Abs. 1 VersAusglG 135

a. Rechtskräftige Entscheidung über den Versorgungsausgleich

b. Gekürzter Versorgungsbezug des Ausgleichspflichtigen 136

c. Kein Versorgungsanspruch des Ausgleichsberechtigten

aa. Grundsätzliche Bedingungen

bb. Strittige Punkte im Rahmen des Tatbestandsmerkmals

(1) Bezug mehrerer Versorgungen

(2) Stellungnahme 140

(3) Deutung des Wortlautes „keine laufende Versorgung erhalten kann" ... 140

(4) Stellungnahme

d. Gesetzlicher Unterhaltsanspruch des Ausgleichsberechtigten

aa. Bestimmung des gesetzlichen Unterhaltsanspruchs 
bb. Unterhaltsanspruch „ohne die Kürzung durch den Versorgungsausgleich" 146

(1) Diskussion bei gesicherter Leistungsfähigkeit des Ausgleichspflichtigen 147

(2) Stellungnahme 148

4. Ergebnis zu $\S 33$ Abs. 1 VersAusglG 149

II. Neueinführung des $\S 33$ Abs. 2 VersAusg|G ................ 150

1. Ausführungen des Gesetzgebers ...................... 150

2. Neue Rechtslage gem. § 33 Abs. 2 VersAusg|G ........... 150

a. Berechnung bei „Neufällen“ ..................... 151

aa. Anknüpfungspunkt des Mindestbetrages .... 152

(1) Meinungsstand ...................... 152

(2) Stellungnahme ....................... 154

bb. Anwendung bei mehreren Anrechten ........ 154

(1) Meinungsstand ...................... 154

(2) Stellungnahme ....................... 155

b. Berechnung bei Verfahren in „Altfällen“ ........... 155

3. Ergebnis ......................................... 157

III. Neueinführung des $\S 33$ Abs. 3 VersAusglG ................ 157

1. Neue Regelung in $\S 33$ Abs. 3 Hs. 1 VersAusglG ......... 158

a. Ausführungen des Gesetzgebers ................. 158

b. Neue Rechtslage gem. $\S 33$ Abs. 3 Hs. 1

VersAusg|G ................................... 158

ad. Unterhaltsanspruch entsprechend dem Unterhaltsbetrag ........................... 160

bb. Unterhaltsanspruch in nicht entsprechender Höhe 163

cc. Bemessung des fiktiven Unterhaltsanspruchs 165

(1) Einbeziehung aller Anrechte bei der Bestimmung 165

(2) Berechnungsbeispiel .................. 166

(3) Stellungnahme ........................ 168 
dd. Berechnung des fiktiven Unterhaltsanspruchs unter Berücksichtigung des Selbstbehalts des Unterhaltspflichtigen 169

(1) Vorgehensweise des AG Waiblingen .... 169

(2) Stellungnahme 172

c. Zwischenergebnis 173

2. Neue Regelung in $\S 33$ Abs. 3 Hs. 2 VersAusglG 174

a. Ausführungen des Gesetzgebers 175

b. Neue Rechtslage gem. $\S 33$ Abs. 3 Hs. 2 VersAusglG: 175

aa. Bei „Neufällen“ 175

bb. Begrenzung in Höhe der beiderseitigen Ausgleichswerte 177

cc. Stellungnahme 178

dd. Einschränkungen gem. $\S 33$ Abs. 3 Hs. 2 VersAusg|G bei „Altfällen“ .................. 179

ee. Stellungnahme ............................ 180

c. Zwischenergebnis .............................. 180

3. Ergebnis zu $\S 33$ Abs. 3 VersAusglG ................... 181

4. Exkurs: Bewertung im verfassungsrechtlichen Kontext 181

a. Verfassungsrechtliche Diskussion hinsichtlich $\S 33$ Abs. 3 VersAusglG .............................. 181

b. Stellungnahme .............................. 182

c. Fazit ..................................... 184

IV. Neueinführung des $\S 33$ Abs. 4 VersAusg|G ................ 184

1. Ausführungen des Gesetzgebers ..................... 185

2. Neue Rechtslage .................................. 185

3. Ergebnis ...................................... 185

B. Verfahrensrechtliche Regelungen gem. $\S 34$ VersAusg|G ....... 186

I. Neue Regelung des $\S 34$ Abs. 1 VersAusg|G ............... 186

1. Ausführungen des Gesetzgebers ...................... 186

2. Neue Rechtslage ................................. 187

a. Einordnung des Anpassungsverfahrens als Familiensache 187 
b. Anpassungsverfahren im Verbund mit der Scheidung? ................................... 189

aa. Zulässige Aufnahme in den Verbund ......... 189

bb. Gegenauffassung .......................... 192

c. Stellungnahme ............................. 192

3. Ergebnis ........................................ 193

II. Neue Regelung des $\S 34$ Abs. 2 VersAusg|G ................. 194

1. Ausführungen des Gesetzgebers ...................... 194

2. Neue Rechtslage ................................. 195

3. Ergebnis ........................................ 196

III. Neueinführung des $\S 34$ Abs. 3 VersAusglG ................ 196

1. Ausführungen des Gesetzgebers ...................... 196

2. Neue Rechtslage .................................... 197

a. Antragstellung und Wirkung .................... 197

b. Zur Leistung von Nachzahlungen .................. 198

3. Ergebnis ........................................... 199

IV. Neueinführung des $\S 34$ Abs. 4 VersAusg|G ................ 199

1. Ausführungen des Gesetzgebers ....................... 199

2. Neue Rechtslage ................................... 199

3. Ergebnis .......................................... 200

V. Neueinführung des $\S 34$ Abs. 5 VersAusg|G ................ 200

1. Ausführungen des Gesetzgebers ...................... 200

2. Neue Rechtslage .................................. 201

3. Ergebnis ....................................... 203

IV. Neueinführung des $\S 34$ Abs. 6 VersAusg|G ................ 204

1. Ausführungen des Gesetzgebers ...................... 204

2. Neue Fassung .................................... 204

a. Abänderung der Anpassung ...................... 204

aa. Eigene Entscheidungsbefugnis des Versorgungsträgers ..................... 205

bb. Notwendigkeit einer familiengerichtlichen Entscheidung ............................ 206

b. Durchführung und Wirkung der Entscheidungen .. 206

3. Ergebnis ........................................ 208

C. Weitere verfahrensrechtliche Besonderheiten ................... 208

I. Fragen zur Beschlussfassung ........................... 208 
II. Probleme im Rahmen der Kostenfestsetzung gem. § 50 FamGKG 209

III. Ergebnis 210

§ 3 Zentrale Probleme innerhalb des Verfahrens gem. §§ 33, 34 VersAusglG 211

A. Eintritt des Rentenfalls vor der Scheidung 211

B. Berechnung des fiktiven Unterhaltsanspruchs bei bestehendem Titel

I. Die Frage der Bindung bei bereits bestehenden Unterhaltstiteln

1. Keine Bindung an bereits bestehende Titel 212

2. Gegenauffassung 215

3. Stellungnahme

II. Fragen zur Ermittlung des gesetzlichen Unterhaltsanspruchs

1. Neuermittlung des Unterhaltsanspruchs im Anpassungsverfahren ............................... 218

2. Gegenauffassung .................................. 220

3. Möglichkeit des Verbunds der Verfahren außerhalb der Scheidung? 221

4. Stellungnahme ...................................... 222

5. Ergebnis .................................... 223

C. Gleichzeitig anhängiges Unterhaltsverfahren bei einem anderen Gericht 224

I. Aussetzung des Unterhaltsverfahrens .................... 224

II. Möglichkeit der Abgabe zu Vermeidung von widersprüchlichen Ergebnissen

1. Abgabe an das für das Versorgungsausgleichsverfahren zuständige Gericht 225

2. Abgabe an das für das Unterhaltsverfahren zuständige Gericht 225

3. Ergebnis 226 


\section{Kapitel 4: Überblick der weiteren unter § 32 VersAusglG fallenden}

Verfahren

§1 Anpassungsverfahren i.S.d. VersAusglG

229

A. Das Anpassungsverfahren gem. $\S \S 35$ und 36 VersAusglG ....... 229

I. Anwendungsfall ..................................... 229

II. Voraussetzungen des $\S 35$ VersAusg|G ..................... 231

III. Durchführung der Anpassung gem. $\S 36$ VersAusg|G ....... 232

B. Das Anpassungsverfahren gem. $\S \S 37$ und 38 VersAusglG ....... 233

I. Anwendungsfall und Vergleich zur alten Rechtslage ......... 233

II. Voraussetzungen für die Anpassung ...................... 236

III. Durchführung der Anpassung gem. § 38 VersAusglG ....... 237

§ 2 Differenzierte Betrachtung bei Abänderungsverfahren ............... 237

A. Die Abänderung gerichtlicher Entscheidungen des Wertausgleichs 238

I. Abgrenzung zu den Verfahren in $\S 227$ FamFG .............. 238

II. Neustrukturierung der Vorschrift § 10a VAHRG a.F. ......... 239

III. Voraussetzungen der Abänderung gem. § 225 FamFG ....... 242

1. Anrecht eines Regelsicherungssystems i.S.v. $§ 32$ VersAusg|G ...................................... 242

2. Änderung des Ausgleichswerts ....................... 242

3. Bewirkung einer wesentlichen Wertänderung gem. $\S 225$ Abs. 3 FamFG

4. Erfüllung einer maßgeblichen Wartezeit gem. § 225 Abs. 4 FamFG .................................... 244

5. Zugunsten eines Ehegatten oder eines Hinterbliebenen ................................. 245

6. Keine Durchführung bei grober Unbilligkeit ............. 245

IV. Durchführung der Abänderung gem. § 226 FamFG ......... 245

B. Zu der Abänderung gerichtlicher Entscheidungen nach $\S \S 51$,

52 VersAusg|G ......................................... 246

I. Zweck der Regelung ..................................... 247

II. Voraussetzungen der Abänderung gem. § 51 VersAusg|G ...247

III. Durchführung der Abänderung gem. § 52 VersAusg|G ...... 249

C. Rechtliche Unterscheidung von Anpassung und Abänderung .... 250 
Kapitel 5: Erfolgreiche Umsetzung der gesetzgeberischen Ziele 253

$\S 1$ Erfolgreiche Umsetzung durch $\S 32$ VersAusglG 253

A. Beseitigung des Gerechtigkeitsdefizits ...................... 253

B. Beseitigung des Anwendungsdefizits ...................... 254

$\S 2$ Erfolgreiche Umsetzung durch $\S \S 33,34$ VersAusglG ................. 255

A. Beseitigung des Gerechtigkeitsdefizits ...................... 255

B. Beseitigung des Anwendungsdefizits .......................256

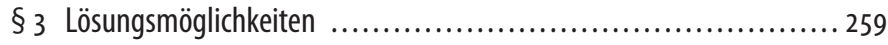

$\S 4$ Gesamtergebnis der Untersuchung .............................. 262

Statistik über Anträge nach $\S \S 4,5,7,8$ VAHRG und $\S \S 33,35,37$

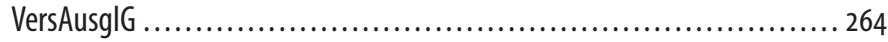


https://doi.org/10.5771/9783828870697-|

Generiert durch IP '172.22.53.54', am 26.04.2023, 10:13:25.

Das Erstellen und Weitergeben von Kopien dieses PDFs ist nicht zulässig. 


\section{Literaturverzeichnis}

Bamberger/Roth, Kommentar zum Bürgerlichen Gesetzbuch, hrsg. von Heinz Georg Bamberger/Herbert Roth, Band 3: $\$ \$ 1297-2385$, EGBGB, 2. Auflage, München 2008 (zitiert: Bamberger/Roth/Bearb., BGB).

Baumbach/Lauterbach/Albers/Hartmann, Zivilprozessordnung, mit Gerichtsverfassungsgesetz und anderen Nebengesetzen, begr. von Adolf Baumbach, fortgesetzt von Wolfgang Lauterbach/Jan Albers/Peter Hartmann und hrsg. von Peter Hartmann, 66. Auflage, München 2008.

-, Zivilprozessordnung, mit FamFG, GVG und anderen Nebengesetzen, begr. von Adolf Baumbach, fort. von Wolfgang Lauterbach/Jan Albers/Peter Hartmann und hrsg. von Peter Hartmann, 74. Auflage, München 2015 (zitiert: Baumbach/ Lauterbach/Albers/Hartmann, ZPO, Aufl.).

Bayer, Walter/Koch, Elisabeth, Aktuelle Fragen des Familienrechts, Baden-Baden 2009 (zitiert: Bayer/Koch).

Beck'scher Online-Kommentar BGB, hrsg. von Heinz Georg Bamberger/Herbert Roth, 40. Auflage, München 2016 (zitiert: Beck'scher Online-Kommentar BGB, Bamberger/Roth/Bearb.).

Bergner, Ludwig, Die Berechnung des Ehezeitanteils einer Beamtenversorgung bei Anwendung von Anrechnungs- oder Ruhevorschriften, NJW 1982, S. 1492 1497 (zitiert: Bergner, NJW 1982).

-, Das Gesetz zur Regelung von Härten im Versorgungsausgleich, DRV 1983, S. 215 - 253 (zitiert: Bergner, DRV 1983).

-, Die Berücksichtigung von Änderungen im Versorgungsausgleich, NJW 1986, S. 217 - 223 (zitiert: Bergner, NJW 1986).

-, Lösungsvorschläge im Zusammenhang mit der Verfassungswidrigkeit des $\$ 1587$ a III BGB und der Barwertverordnung, FamRZ 1999, S. $1487-1493$ (zitiert: Bergner, FamRZ 1999).

-, Einwände gegen den Entwurf einer Dritten Verordnung zur Änderung der Barwert-VO und Vorschläge für zweckmäßigere Übergangsregelungen, FPR 2006, S. 55 - 63 (zitiert: Bergner, FPR 2006).

-, Strategien im Versorgungsausgleich - Lösung der vorhandenen tatsächlichen Probleme bis zur Reform, FPR 2007, S. 142 - 151 (zitiert: Bergner, FPR 2007).

-, Der reformierte Versorgungsausgleich - Die wichtigsten Neuerungen des materiellen Rechts, NJW 2009, S. 1169 - 1175. 
-, Der reformierte Versorgungsausgleich, Verfahrensrecht, Übergangsrecht und anzuwendendes altes bzw. neues Recht, NJW 2009, S. 1233 - 1237 (zitiert: Bergner, NJW 2009).

-, Anwendung und Nichtanwendung der Bagatellklausel des $\$ 18$ VersAusglG, NJW 2010, S. 3269 - 3274.

-, Das Unterhaltsprivileg der $\$ \$ 33,34$ VersAusglG, NJW 2010, S. 3545 - 3547 (zitiert: Bergner, NJW 2010).

-, Das Unterhaltsprivileg, FPR 2011, S. 483 - 490 (zitiert: Bergner, FPR 2011).

Bergner, Ludwig/Borth, Helmut, Das Unterhaltsprivileg nach $\$_{33}$ VersAusglG Verfassungsmäßigkeit der Beschränkung auf Regelsicherungssysteme sowie inhaltliche Reichweite -, FamRZ 2013, S. 589 - 593 (zitiert: Bergner/Borth, FamRZ 2013).

Berliner Kommentare, FamFG, Gesetz über das Verfahren in Familiensachen und in Angelegenheiten der freiwilligen Gerichtsbarkeit, hrsg. von Dirk Bahrenfuss, Berlin 2009 (zitiert: Berliner Kommentar/Bearb., FamFG).

$B G B-R G R K$, Das Bürgerliche Gesetzbuch mit besonderer Berücksichtigung der Rechtsprechung des Reichsgerichts und des Bundesgerichtshofes, Kommentar, hrsg. von Bernhard Böckermann/Anneliese Cuny/Karin Graßhof/Elmar Kalthoener/Friedrich Lohmann/Hartmut Wick, 12. Auflage, Berlin 1999 (zitiert: BGB - RGRK/Bearb.).

Bork/Jacoby/Schwab, FamFG Kommentar, hrsg. von Reinhard Bork/Florian Jacoby/Dieter Schwab, 2. Auflage, München 2013 (zitiert: Bork/Jacoby/Schwab/Bearb., FamFG).

Borth, Helmut, Der Versorgungsausgleich: in anwaltlicher und familiengerichtlicher Praxis, 4. Auflage, Köln 2008.

-, Der Versorgungsausgleich: in anwaltlicher und familiengerichtlicher Praxis, 6. Auflage, Köln 2012.

-, Der Versorgungsausgleich: in anwaltlicher und familiengerichtlicher Praxis, 7. Auflage, Köln 2014 (zitiert: Borth, Versorgungsausgleich, Aufl.).

-, Aktuelle Probleme des reformierten Versorgungsausgleichs, FamRZ 2010, S. 1210 - 1216 (zitiert: Borth, FamRZ 2010).

-, Anmerkung zur Entscheidung des OLG Hamm - VersAusglG $\$ \$ 33$, 34; FamFG $\$ \$$ 238, 239; ZPO \$ 767, FamRZ 2011, S. 815 - 818 (zitiert: Borth, FamRZ 2011).

Brudermüller, Gerd, Die Entwicklung des Familienrechts seit Mitte 2011 - Güterrecht und Versorgungsausgleich, NJW 2012, S. 1266 - 1271 (zitiert: Brudermüller, NJW 2012).

Bumiller/Harders/Schwamb, FamFG, Freiwillige Gerichtsbarkeit, hrsg. von Ursula Bumiller/Dirk Harders/Werner Schwamb, 11. Auflage, München 2015 (zitiert: Bumiller/Harders/Schwamb, FamFG).

Cuny, Annelise, Unterhalt und Versorgungsausgleich der geschiedenen Ehefrau, Beiträge zur Reform des Ehescheidungsrechts, Frankfurt am Main, Berlin 1970 (zitiert: Cuny). 
Dastmaltchi, Elisabeth, Das formelle Scheidungsverfahren nach geltendem Recht und nach der geplanten Änderung des familiengerichtlichen Verfahrens, FPR 2007, S. 226 - 231 (zitiert: Dastmaltchi, FPR 2007).

Deisenhofer, Ulrich, Die Zusatzversorgung des öffentlichen Dienstes und $\$ 32$ VersAusglG, FamRZ 2011, S. 1122 - 1123 (zitiert: Deisenhofer, FamRZ 2011).

Deutscher Familiengerichtstag e.V., Strukturreform des Versorgungsausgleichs, Stellungnahme des Deutschen Familiengerichtstages e. V. und der Wissenschaftlichen Vereinigung für Familienrecht e.V. zu dem Abschlussbericht der hierfür eingesetzten Regierungskommission v. 27.10.2004, FamRZ 2005, S. 852 - 856 (zitiert: Deutscher Familiengerichtstag e.V., FamRZ 2005).

Diederichsen, Uwe, Das Recht der Ehescheidung nach dem 1. EheRG (Scheidungsgründe), NJW 1977, S. 273 - 279 (zitiert: Diederichsen, NJW 1977).

DIW Berlin, Erwerbstätigkeit von Frauen - Entwicklung der Frauenerwerbsquote, http://de.statista.-com/statistik/daten/studie/13541/umfrage/frauenerwerbsquote---entwicklung-der-erwerbstaetig-keit-von-frauen/ (3.10.2016, 10.05 Uhr) (zitiert: DIW Berlin, Frauenerwerbsquote).

Dörr, Claus, Abänderungsverfahren nach $\$ 1$ oa VAHRG, FPR 2007, S. 130 - 133 (zitiert: Dörr, FPR 2007).

Drochner, Sabine/Uebelhack, Birgit, Die Betriebsrente im Versorgungsausgleich Textsammlung, mit Auszug aus der Amtlichen Begründung und einer Einführung von Prof. Dr. Franz Ruland, hrsg. von der Arbeitsgemeinschaft für betriebliche Altersversorgung e.V., 3. Auflage, Heidelberg 2014 (zitiert: Drochner/ Uebelhack, Betriebsrente im Versorgungsausgleich).

Eichenhofer, Eberhard, Drei Wege zum Versorgungsausgleich, FamRZ 2008, S. 950 - 953 (zitiert: Eichenhofer, FamRZ 2008).

-, Stellungnahme zum Entwurf eines VA-Strukturreformgesetzes (VAStrRefG) BT-Drucks. 16/10144 - aus Anlass der Sitzung des Rechtsausschusses des Deutschen Bundestages am 3.12.2008, http://webarchiv.bundestag.de/cgi/show.php? fileToLoad=1090\&id=1118 (3.10.2016 10.41 Uhr) (zitiert: Eichenhofer, Stellungnahme).

-, Nachträgliche Änderung des Versorgungsausgleichs wegen Todes des Ausgleichsberechtigten vor Eintritt des Leistungsfalles, FamFR 2012, S. 108 - 109 (zitiert: Eichenhofer, FamFR 2012).

Eichenhofer/Rische/Schmähl, Handbuch der gesetzlichen Rentenversicherung SGB VI, hrsg. von Eberhard Eichenhofer/Herbert Rische/Winfried Schmähl, 2. Auflage, Köln 2012 (zitiert: Handbuch d. ges. RV/Bearb.).

Erman, Bürgerliches Gesetzbuch, Handkommentar mit AGG, EGBGB (Auszug), ErbbauRG, LPartG, ProdHaftG, UKlaG, VBVG, VersAusglG und WEG, hrsg. von Harm Peter Westermann/Barbara Grunewald/Georg Maier-Reimer, Band II, 14. Auflage, Köln 2014 (zitiert: Erman/Bearb., BGB).

Fest, Timo, Zwei Jahre Reform des familiengerichtlichen Verfahrens: Eine Zwischenbilanz, NJW 2011, S. 2611 - 2615 (zitiert: Fest, NJW 2011). 
Friederici, Peter, Anmerkungen zur Strukturreform des Versorgungsausgleichs, Forum Familienrecht 2005, S. 140 - 143 (zitiert: Friederici, Forum Familienrecht 2005).

-, Praxis des Versorgungsausgleichs, Köln 2011 (zitiert: Friederici, Praxis des Versorgungsausgleichs).

Garbe/Ullrich, NomosProzessHandbuch, Verfahren in Familiensachen, FamFG/ ZPO/ BGB, hrsg. von Roland Garbe/Christoph Ullrich, 3. Auflage, Baden-Baden 2012 (zitiert: Garbe/Ullrich/Bearb., Verfahren in Familiensachen).

Gerhardt/v. Heintschel-Heinegg/Klein, Handbuch des Fachanwalts, Familienrecht, hrsg. von Peter Gerhardt/Bernd von Heintschel-Heinegg/Michael Klein, 9. Auflage, München 2013 (zitiert: Gerhardt/v. Heintschel-Heinegg/Klein/Bearb., Familienrecht).

Gernhuber, Joachim/Coester-Waltjen, Dagmar, Familienrecht, 5. Auflage, München 2006.

-, Familienrecht, 6. Auflage, München 2010 (zitiert: Gernhuber/Coester-Waltjen, Familienrecht, Aufl.).

Glockner/Hoenes/Weil, Der neue Versorgungsausgleich, hrsg. von Rainer Glockner/Ute Hoenes/ Arndt Voucko-Glockner/Klaus Weil, München 2009 (zitiert: Glockner/Hoenes/Weil, Der neue Versorgungsausgleich).

Götsche, Frank, Das Unterhaltsprivileg im reformierten Versorgungsausgleich, ZFE 2010, S. 407 - 414 (zitiert: Götsche, ZFE 2010).

Götz, Isabell, Das neue Familienverfahrensrecht - Erste Praxisprobleme, NJW 2010, S. 897 - 902 (zitiert: Götz, NJW 2010).

-, Grenzen richterlicher Rechtsfortbildung im nachehelichen Unterhaltsrecht, NJW 2011, S. 801 -808 (zitiert: Götz, NJW 2011).

Gutdeutsch, Werner, Das Unterhaltsprivileg nach neuem Recht, FamRB 2010, S. 149 - 155 (zitiert: Gutdeutsch, FamRB 2010).

-, Das Unterhaltsprivileg im Verbund, FamRZ 2010, S. 1140 - 1141 (zitiert: Gutdeutsch, FamRZ 2010).

Gutdeutsch, Werner/Lardschneider, Ullrich, Probleme der neuen Ausgleichsform des Gesetzes zur Regelung von Härten im Versorgungsausgleich, FamRZ 1983, S. 845 - 851 (zitiert: Gutdeutsch/Lardschneider, FamRZ 1983).

Häußermann, Röse, Zehn Fallstricke des neuen Versorgungsausgleichs, FPR 2009, S. 223 - 231 (zitiert: Häußermann, FPR 2009).

Hahne, Meo-Micaela, Stellungnahme zum Entwurf eines VA-Strukturreformgesetzes (VAStrRefG) - BT-Drucks. 16/10144 - aus Anlass der Sitzung des Rechtsausschusses des Deutschen Bundestages am 3.12.2008, http://webarchiv.bundes tag.de/cgi/show.php?fileToLoad =1090\&id=1118 (3.10.2016, 11.28 Uhr) (zitiert: Hahne, Stellungnahme).

Hauß, Jörg, Der neue Versorgungsausgleich, AnwBl 2009, S. 577 - 581 (zitiert: Hauß, AnwBl 2009). 
-, Praktische Fragen des neuen Versorgungsausgleichs, FamRB 2010, S. 251 - 257 (zitiert: $\operatorname{Hau} \beta$, FamRB 2010).

Haußleiter, FamFG, Gesetz über das Verfahren in Familiensachen und in den Angelegenheiten der freiwilligen Gerichtsbarkeit, Kommentar, hrsg. von Martin Haußleiter, München 2011 (zitiert: Haußleiter/Bearb., FamFG).

Heiß, Thomas A., Rentenkürzung und Unterhaltsprivileg nach $\$ \$ 33,34$ VersAusglG - Aussetzung der Rentenkürzung trotz Wegfall des Rentner-/ Pensionistenprivilegs, FamFR 2011, S. 291 - 294 (zitiert: Heiß, FamFR 2011).

Heubeck, Klaus, „Versicherungsmathematische Gesichtspunkte bei der Strukturreform des Versorgungsausgleichs“ - Vorabzusammenfassung vom 29. August 2003, Abschlussbericht der Kommission Strukturreform des Versorgungsausgleichs, Anhang II Dokumente, http://www.gesmat.bundesgerichtshof.de/gesetzesmaterialien/16_wp/vastrrefg/Anhang_Abschlussbericht.pdf

(3.12.2016 11.32 Uhr) (zitiert: Heubeck, Abschlussbericht der Kommission Strukturreform des Versorgungsausgleichs).

Holzwarth, Andreas, Die Übergangsvorschriften nach dem Entwurf der Bundesregierung für ein Gesetz zur Strukturreform des Versorgungsausgleichs, FamRZ 2008, S. 2168 - 2174 (zitiert: Holzwarth, FamRZ 2008).

-, Rechtsprechungsübersicht zum reformierten Versorgungsausgleich, FamRZ 2011, S. 933 - 945 (zitiert: Holzwarth, FamRZ 2011).

Hoppenz, Familiensachen, hrsg. von Rainer Hoppenz, 9. Auflage, Heidelberg 2009 (zitiert: Hoppenz/Bearb., Familiensachen).

Horndasch/Viefhues, FamFG - Kommentar zum Familienverfahrensrecht, Betreuungs- und Unterbringungssachen, Nachlass- und Teilungssachen, Anwaltsgebühren und Gerichtskosten, hrsg. von K.-Peter Horndasch/Wolfram Viefhues, 2. Auflage, Münster 2010 (zitiert: Horndasch/Viefhues/Bearb., FamFG).

Jarass/Pieroth, GG, Grundgesetz für die Bundesrepublik Deutschland, Kommentar, hrsg. von Hans D. Jarass/Bodo Pieroth, 14. Auflage, München 2016 (zitiert: Jarass/Pieroth/Bearb., GG).

Johannsen/Henrich, Eherecht, Trennung, Scheidung, Folgen, Kommentar, hrsg. von Dieter Henrich, 4. Auflage, München 2003 (zitiert: Johannsen/Henrich/Bearb., Eherecht, Aufl.).

-, Familienrecht, Scheidung, Unterhalt, Verfahren, Kommentar, hrsg. von Dieter Henrich/Kurt H. Johannsen/Christof Althammer/Gerd Brüdermüller/Dieter Büte/Isabell Götz/Hans-Ulrich /Dieter Henrich/Meo-Micaela Hahne/Dieter Henrich/Andreas Holzwarth/Wolfgang Jaeger/Winfried Maier/Angelika Markwardt, 5. Auflage, München 2010.

-, Familienrecht, Scheidung, Unterhalt, Verfahren, Kommentar, hrsg. von Dieter Henrich/Kurt H. Johannsen/Christof Althammer/Gerd Brüdermüller/Dieter Büte/Isabell Götz/Monika Hamm/Eckart Hammermann/Dieter Henrich/ Andreas Holzwarth/Wolfgang Jaeger/Winfried Maier/Angelika Markwardt, 6. Auflage, München 2015 (zitiert: Johannsen/Henrich/Bearb., Familienrecht, Aufl.). 
juris PraxisKommentar, BGB, Familienrecht, Band 4, hrsg. von Wolfram Viefhues/ Maximilian Herberger/Michael Martinek/Helmut Rüßmann/Stephan Weth, 5. Auflage, Saarbrücken 2011 (zitiert: jurisPK/Bearb., Familienrecht).

Keidel, FamFG, Kommentar zum Gesetz über das Verfahren in Familienverfahren und in den Angelegenheiten der Freiwilligen Gerichtsbarkeit, begr. von Theodor Keidel, hrsg. von Helmut Engelhardt/Werner Sternal, 18. Auflage, München 2014 (Keidel/Bearb., FamFG).

Kemper, Rainer, Versorgungsausgleich in der Praxis, ZAP Verlag 2011 (zitiert: Kemper, Versorgungsausgleich in der Praxis).

-, FamFG/ FGG/ ZPO, Kommentierte Synopse, 2. Auflage, Baden-Baden 2009 (zitiert: Kemper, FamFG).

-, Die Abänderung von Altentscheidungen zum Versorgungsausgleich, FuR 2010, S. 189 - 197 (zitiert: Kemper, FuR 2010).

-, Der neue Versorgungsausgleich, ZFE 2009, S. 204 - 211 (zitiert: Kemper, ZFE 2009).

Keske, Monika, Das neue FamGKG, Kurzkommentar mit Synopse, Köln 2009 (zitiert: Keske, FamGKG).

Klauser, Karl-August, Die (vorläufige) Korrektur des Versorgungsausgleichs, MDR 1983, S. 529-534 (zitiert: Klauser, MDR 1983).

Koch, Handbuch des Unterhaltsrechts, begr. von Wolfgang Köhler, hrsg. Elisabeth Koch, 12. Auflage, München 2012 (zitiert: HdU/Bearb.).

Kommission "Strukturreform des Versorgungsausgleichs", Abschlussbericht, www.dnoti.de/DOC/2007/Abschlussbericht.pdf (3.10.2016, $12.26 \mathrm{Uhr})$ (zitiert: Kommission Strukturreform des Versorgungsausgleichs, Abschlussbericht).

Koritz, Nikola, Das neue FamFG, München 2009 (zitiert: Koritz, Das neue FamFG).

Kraus, Cornelia, Grundlagen des Unterhaltsrechts, Überlegungen für eine Harmonisierung des Verwandten-, Betreuungs- und Geschiedenenunterhalts, BadenBaden 2011 (zitiert: Kraus).

Kreikebohm, Ralf, Sozialgesetzbuch, Gesetzliche Rentenversicherung - SGB VI -, 4. Auflage, München 2013 (zitiert: Kreikebohm/Bearb.).

Krenzler, Michael/Borth, Helmut Anwaltshandbuch Familienrecht, 2. Auflage, Köln 2012 (zitiert: Krenzler/Borth/Bearb.).

Lüderitz, Alexander/Dethloff, Nina, Familienrecht, Ein Studienbuch, 28. Auflage, München 2007 (zitiert: Lüderitz/Dethloff, Familienrecht).

Maier, Kurt/Michaelis, Klaus, Versorgungsausgleich, in der Rentenversicherung, hrsg. von der Bundesversicherungsanstalt für Angestellte, 6. Auflage, Berlin 2000 (zitiert: Maier/Michaelis, Versorgungsausgleich).

Michaelis, Klaus, Berücksichtigung von Rechtsänderungen bei der Bewertung von Anwartschaften der gesetzlichen Rentenversicherung im Versorgungsausgleich, FamRZ 1985, S. 550 - 554 (zitiert: Michaelis, FamRZ 1985). 
Müller, Tanja, Anwendbarkeit des $\$ 5$ I VAHRG bei der Möglichkeit des Rentenbezuges durch den ausgleichsberechtigten Ehegatten?, FamRZ 2005, S. 1721 1725 (zitiert: Müller, FamRZ 2005).

Münchener AnwaltsHandbuch, Familienrecht, hrsg. von Klaus Schnitzler, 4. Auflage, München 2014 (zitiert: MAH/Bearb., Familienrecht).

Münchener Kommentar, zum Bürgerlichen Gesetzbuch, Versorgungsausgleich, Fassung vor dem 1. September 2009, hrsg. von Franz Jürgen Säcker/Roland Rixecker/Kurt Rebmann, Band 7: Familienrecht I, $\$ \$ 1297-1588$, VAHRG, VAÜG, HausratsV, 5. Auflage, München 2010.

-, zum Bürgerlichen Gesetzbuch, hrsg. von Franz Jürgen Säcker/Roland Rixecker/ Hartmut Oetker/Bettina Limperg, Band 7: Familienrecht I, \$\$1297 - 1588, VersAusglG, GewSchG, LPartG, 6. Auflage, München 2013 (zitiert: MK/Bearb., BGB, Aufl.).

Muscheler, Karlheinz, Familienrecht, 3. Auflage, München 2013 (zitiert: Muscheler, Familienrecht).

Musielak/Borth, Familiengerichtliches Verfahren Kommentar, 1. und 2. Buch, hrsg. von Helmut Borth/Mathias Grandel/Hans-Joachim Musielak, 5. Auflage, München 2015 (zitiert: Musielak/Borth/Bearb., FamFG).

Naegele, Wolfgang, Der Versorgungsausgleich, Grundlagen - Probleme - Dispositionsmöglichkeiten, 3. Auflage, Freiburg 1990 (zitiert: Naegele, Der Versorgungsausgleich).

Niepmann, Birgit/Schwamb, Dieter, Die Entwicklung des Unterhaltsrechts seit Mitte 2009, NJW 2010, S. 2400 - 2408 (zitiert: Niepmann/Schwamb, NJW 2010).

NomosFormulare, Familienrecht, Vereinbarungen, Verfahren, außergerichtliche Kompetenzen, hrsg. von Karin Meyer-Götz, 3. Auflage, Baden-Baden 2014 (zitiert: Meyer-Götz/Bearb.).

NomosKommentar, Bürgerliches Gesetzbuch Handkommentar, hrsg. von Heinrich Dörner/Ina Ebert/Thomas Hoeren/Rainer Kemper/Ingo Saenger/Klaus Schreiber/Hans Schulte-Nötke/Reiner Schulze/Ansgar Staudinger, 5. Auflage, BadenBaden 2007 (zitiert: HK/Bearb., BGB).

-, Bürgerliches Gesetzbuch Familienrecht Handkommentar, hrsg. von Dagmar Kaiser/Klaus Schnitzler/Peter Friederici/Roger Schilling, 3. Auflage, Baden-Baden 2014 (zitiert: HK/Bearb., BGB Familienrecht).

-, Familienrecht Handkommentar, hrsg. von Werner Schulz/Jörn Hauß, Baden-Baden 2008 (zitiert: HK/Bearb., Familienrecht).

-, Familienrecht Handkommentar, hrsg. von Werner Schulz/Jörn Hauß, 2. Auflage, Baden-Baden 2011 (zitiert: HK/Bearb., Familienrecht, Aufl.).

-, Familienverfahrensrecht Handkommentar, hrsg. von Rainer Kemper/Klaus Schreiber, 3. Auflage, Baden-Baden 2015 (zitiert: HK/Bearb., FamFG).

-, Sozialgesetzbuch SGB VI, Gesetzliche Rentenversicherung, Lehr- und Praxiskommentar, hrsg. Helmut Reinhardt, Baden-Baden 2006 (zitiert: HK/Bearb., SGB VI). 
-, Versorgungsausgleichsrecht, VersAusglG, BGB, FamFG, FamGKG VersAusglKassG, BVersTG, SGB VI, Handkommentar, hrsg. Frank Götsche/Frank Rehbein/Christian Breuers, 2. Auflage, Baden-Baden 2012 (zitiert: HK/Bearb., Versorgungsausgleich).

Palandt, Otto, Bürgerliches Gesetzbuch mit Nebengesetze, insbesondere mit Einführungsgesetz (Auszug), Allgemeines Gleichbehandlungsgesetz (Auszug), BGB-Informationspflichten-Verordnung, Unterlassungsklagengesetz, Produkthaftungsgesetz, Erbbaurechtsverordnung, Wohnungseigentumsgesetz, Hausratsverordnung, Vormünder- und Betreuervergütungsgesetz, Lebenspartnerschaftsgesetz, Gewaltschutzgesetz (Artikel 1), 68. Auflage, München 2009.

-, Bürgerliches Gesetzbuch mit Nebengesetzen, insbesondere mit Einführungsgesetz (Auszug) einschließlich Rom I- und Rom II- Verordnungen, Allgemeines Gleichbehandlungsgesetz (Auszug), BGB-Informationspflichten- Verordnung, Wohn- und Betreuungsvertragsgesetz, Unterlassungsklagengesetz, Produkthaftungsgesetz, Erbbaurechtsgesetz, Wohnungseigentumsgesetz, Versorgungsausgleichsgesetz, Lebenspartnerschaftsgesetz, Gewaltschutzgesetz, 75. Auflage, München 2016 (zitiert: Palandt/Bearb., BGB, Aufl.).

Prütting/Helms, FamFG Kommentar, Gesetz über das Verfahren in Familiensachen und in den Angelegenheiten der freiwilligen Gerichtsbarkeit mit Gesetz über Gerichtskosten in Familiensachen, hrsg. von Hanns Prütting/Tobias Helms, 3. Auflage, Köln 2013 (zitiert: Prütting/Helms/Bearb., FamFG).

Prütting/Wegen/Weinreich, BGB Kommentar, hrsg. von Hanns Prütting/Gerhard Wegen/Gerd Weinreich, 4. Auflage, Köln 2009.

-, BGB Kommentar, hrsg. von Hanns Prütting/Gerhard Wegen/Gerd Weinreich, 10. Auflage, Köln 2015 (zitiert: PWW/Bearb., BGB, Aufl.).

Rauscher, Thomas, Familienrecht, 2. Auflage, Heidelberg 2008 (zitiert: Rauscher, Familienrecht).

Rehme, Eckhard, Verfassungswidrigkeit des Entwurfs einer Dritten Verordnung zur Änderung der Barwert-Verordnung, FuR 2006, S. 112 - 116 (zitiert: Rehme, FuR 2006).

-, Rechtliche und rechtspolitische Bedenken gegen das Ausgleichskonzept im Diskussionsentwurf des Bundesministeriums der Justiz v. 29.8.2007 für ein Gesetz zur Strukturreform des Versorgungsausgleichs, FamRZ, 2008, S. $738-747$ (zitiert: Rehme, FamRZ 2008).

Ruland, Franz, Versorgungsausgleich, Ausgleich, steuerliche Folgen und Verfahren, NJW Praxis, Band 28, 3. Auflage, München 2011.

-, Versorgungsausgleich, Ausgleich, steuerliche Folgen und Verfahren, NJW Praxis, Band 28, 4. Auflage, München 2015 (zitiert: Ruland, Versorgungsausgleich, Aufl.).

-, Die Durchführung des Wertausgleichs, FPR 2011, S. 479 - 482 (zitiert: Ruland, FPR 2011). 
-, Verfassungswidrigkeit des $\$ 32$ VersAusglG, FamFR 2012, S. 314 - 316 (zitiert: Ruland, FamFR 2012).

Sachs, Grundgesetz Kommentar, hrsg. von Michael Sachs, 7. Auflage, München 2014 (zitiert: Sachs/Bearb., GG).

Schlüter, Wilfried, Familienrecht, 14. Auflage, Heidelberg [München, Landsberg, Frechen, Hamburg] 2013 (zitiert: Schlüter, Familienrecht).

Schmidbauer, Wilhelm, Der Versorgungsausgleich - Die Reform - Eine Einführung und ausführliche Erläuterung aufgrund amtlicher Unterlagen des neuen vom 1. September 2009 geltenden Rechts, z.T. grundlegend geänderten Rechts, 8. Auflage, Münsing 2009 (zitiert: Schmidbauer, Der Versorgungsausgleich).

Schmidt-Bleibtreu/Hofmann/Hopfauf, GG, Grundgesetz Kommentar, begr. von Bruno Schmidt-Bleibtreu/Franz Klein, hrsg. von Hans Hofmann/Axel Hopfauf, 12. Auflage, Köln, München 2011 (zitiert: Schmidt-Bleibtreu/Hofmann/Hopfauf/Bearb., GG).

Schramm, Barbara, Unbilligkeitsaufwand im VA-Abänderungsverfahren, NJWSpezial 2007, S. 199 - 200 (zitiert: Schramm, NJW-Spezial 2007).

Schulte-Brunert, Kai, Das neue FamFG, 2. Auflage, Köln 2010 (zitiert: Schulte-Brunert, Das neue FamFG).

Schulte-Brunert/Weinreich, Kommentar des FamFG, hrsg. von Kai Schulte-Brunert/Gerd Weinreich, 4. Auflage Köln 2013 (zitiert: Schulte-Brunert/Weinreich/ Bearb., FamFG).

Schwab, Dieter, Familienrecht, Verfassungswidrigkeit des Versorgungsausgleichs?, FamRZ 1977, S. 768 - 773 (zitiert: Schwab, FamRZ 1977).

-, Familienrecht, 16. Auflage, München 2008.

-, Familienrecht, 24. Auflage, München 2016 (zitiert: Schwab, Familienrecht, Aufl.).

Schwamb, Werner, Erste Rechtsprechung zum Unterhaltsprivileg gem. \$\$33, 34 VersAusglG, NJW 2011, S. 1648 - 1651 (zitiert: Schwamb, NJW 2011).

Sethe, Rolf/Höland, Armin, Versorgungsausgleich, Schriften zum Notarrecht, hrsg. von der Deutschen Notarrechtlichen Vereinigung e.V. Sachsen-Anhalt, BadenBaden 2011 (zitiert: Sethe/Höland/Bearb. Versorgungsausgleich).

Simon, Carola, Die Berücksichtigung von aus einer Nachzahlung resultierender Rentenanwartschaft im Versorgungsausgleich, Bern Schweiz 2003 (zitiert: Simon).

Sodan, Grundgesetz, Beck'scher Kompakt-Kommentar, hrsg. von Helge Sodan, 3. Auflage, München 2015 (zitiert: Sodan/Bearb., GG).

Soergel, Bürgerliches Gesetzbuch mit Einführungsgesetz und Nebengesetzen, begründet von Hs. Th. Soergel, neu hrsg. von W. Siebert/, Band 18, Familienrecht 2 (\$\$1587-1588) VAHRG, VAÜG, 13. Auflage, Stuttgart 2000 (zitiert: Soergel/ Bearb., BGB). 
Staudinger, Kommentar zum Bürgerlichen Gesetzbuch mit Einführungsgesetz und Nebengesetzen, Buch 4 Familienrecht, $\$ \$ 1587$ - 1588 (inkl. Barwert-VO), VAHRG (Versorgungsausgleich), Neubearbeitung, Berlin 2004 (zitiert: Staudinger/Bearb., BGB).

Tschernitschek, Horst/Saar, Stefan Familienrecht Lehrbuch, 4. Auflage, München 2008 (zitiert: Tschernitschek/Saar, Familienrecht).

Weinreich/Klein, Fachanwaltskommentar Familienrecht, BGB, VersAusglG, EGBGB (Auszüge), LPartG, GewSchG, EStG (Auszüge), hrsg. von Gerd Weinreich/Michael Klein, 5. Auflage, Köln 2013 (zitiert: Weinreich/Klein/Bearb., Familienrecht).

Wick, Hartmut, Der neue Versorgungsausgleich in der Praxis: erste Erfahrungen mit dem neuen Recht, Bonn 2009, (zitiert: Wick, Der neue Versorgungsausgleich in der Praxis).

-, Die Rechtsprechung zum neuen Versorgungsausgleich, Teil 1, FuR 2011, S. 363 369.

-, Die Rechtsprechung zum neuen Versorgungsausgleich, Teil 2, FuR 2011, S. 436 440.

-, Die Rechtsprechung zum neuen Versorgungsausgleich, Teil 3, FuR 2011, S. 555 561.

-, Die Rechtsprechung zum neuen Versorgungsausgleich, Teil 4, FuR 2011, S. 605 611, (zitiert: Wick, FuR 2011).

Zimmermann, Walter, Das neue FamFG, Verfahrensrecht, Rechtsmittel, Familiensachen, Betreuung, Unterbringung, Nachlasssachen und Kosten, 2. Auflage, München 2011 (zitiert: Zimmermann, Das neue FamFG). 